Vol. 54 (1996) [363-368]

\title{
ON CLOSED RADICAL ORBITS IN HOMOGENEOUS COMPLEX MANIFOLDS
}

\author{
Bruce Gilligan
}

\begin{abstract}
Suppose $G$ is a complex Lie group having a finite number of connected components and $H$ is a closed complex subgroup of $G$ with $H^{\circ}$ solvable. Let $R_{G}$ denote the radical of $G$. We show the existence of closed complex subgroups $I$ and $J$ of $G$ containing $H$ such that $I / H$ is a connected solvmanifold with $I^{\circ} \supset R_{G}$, the space $G / J$ has a Klein form $S_{G} / A$, where $A$ is an algebraic subgroup of the semisimple complex Lie group $S_{G}:=G / R_{G}$, and, unless $I=J$, the space $J / I$ has Klein form $\widehat{S} / \widehat{\Gamma}$, where $\widehat{\Gamma}$ is a Zariski dense discrete subgroup of some connected positive dimensional semisimple complex Lie group $\widehat{S}$.
\end{abstract}

\section{INTRODUCTION}

Methods which allow one to study quotients of Lie groups by discrete subgroups by considering quotients of solvable and semisimple Lie groups separately are very helpful. One such method is presented in [1, Theorem 1] for $G / \Gamma$ compact, where $\Gamma$ is a discrete subgroup of a Lie group $G$. Also decompositions of compact real homogeneous spaces have been introduced by Gorbatsevich. He developed what he calls the natural and structure bundles, where these involve double coset spaces, for example, see the fifth chapter in the book [6] for their descriptions. In the case of a quotient of complex Lie groups one would like to have a decomposition which is compatible with the complex structure.

In order to get a decomposition we consider the natural map $\pi: G \rightarrow S_{G}:=G / R_{G}$, where $R_{G}$ denotes the radical of $G$. For arbitrary $G / H$ difficulties arise because $\pi(H)$ need not be closed in $S_{G}$. To handle such problems we use a result of ZassenhausAuslander, extended in Theorem $A$ in the Appendix of [11], which is applicable whenever $H^{\circ}$, the connected component of the identity of $H$, is solvable. It follows that $U^{\circ}$, the connected component of the identity of the closure of $\pi(H)$, is solvable. The normaliser $N$ in $S_{G}$ of the complexification $\tilde{U}$ of $U^{\circ}$ is an algebraic group containing

Received 4th December, 1996.

We would like to thank D. N. Akhiezer for helpful discussions concerning this work. Also we are grateful for support as a visitor under the Abstract Analysis and Geometry Program at The Australian National University, Canberra, where part of the work on this paper was completed.

Copyright Clearance Centre, Inc. Serial-fee code: 0004-9729/96 \$A2.00+0.00. 
$\pi(H)$ which cannot be semisimple. If $N$ is solvable, then $J:=\pi^{-1}(N)$ is a closed complex subgroup of $G$ containing $H$ with $J^{\circ}$ solvable such that $G / J$ has a Klein form $S_{G} / A$, where $A$ is an algebraic subgroup of the semisimple complex Lie group $S_{G}$. If $N$ is not solvable, one proceeds by recursion and, if at some stage there is a normaliser which is solvable, one is in a situation similar to the one described above. Otherwise, because the dimension of $G / H$ is finite, there is a closed complex subgroup $J_{k}$ of $G$ containing $H$ such that the orbits of the radical of $J_{k}$ are closed in $J_{k} / H$. It is then easy to show that there exist closed complex subgroups $I$ and $J$ of $G$ containing $H$ such that $I / H$ is a connected complex solvmanifold, $G / J$ has a Klein form $S_{G} / A$ with $A$ an algebraic subgroup of $S_{G}$, and, unless $I=J$, the space $J / I$ has a Klein form $\widehat{S} / \widehat{\Gamma}$, where $\widehat{\Gamma}$ is a Zariski dense discrete subgroup of a semisimple complex group $\widehat{S}$. This generalises a fibration for homogeneous complex manifolds with discrete isotropy which can be found in [4, Theorem 2]. But explicit Klein forms are not given in that result, as they are here.

Now any solvmanifold (respectively, quotient of algebraic groups) admits the structure of a vector bundle over a compact manifold, see [2] or [10] (respectively, [9] or [8]). Thus complicated topological problems concerning $G / H$ with $H^{\circ}$ solvable reduce to questions about the $\widehat{S} / \widehat{\Gamma}$ corresponding to $J / I$. For example, this idea was applied in [5] to homogeneous complex manifolds with more than two ends in order to give an explicit decomposition of these spaces that shows "essentially where their ends live".

Throughout we will denote the connected component of the identity of a complex Lie group $L$ by $L^{\circ}$ and its radical by $R_{L}$. Let $\pi_{L}: L \rightarrow L / R_{L}$ be the natural map.

\section{THE STRUCTURE THEOREM}

Theorem 1. Suppose $X=G / H$ is a connected homogeneous complex manifold, where $G$ is a complex Lie group which has a finite number of connected components and is not solvable and $H$ is a closed complex subgroup of $G$ with $H^{\circ}$ solvable. Then there exist closed complex subgroups $I$ and $J$ of $G$ containing $H$ such that $I^{\circ}$ is solvable, $I^{\circ} \supset R_{G}$, and $I / H$ is connected, and $J$ has a finite number of connected components with $G / J$ having a Klein form $S_{G} / A$, where $A$ is an algebraic subgroup of the semisimple complex Lie group $S_{G}:=G / R_{G}$. Moreover, unless $I=J$, the space $J / I=\widehat{S} / \widehat{\Gamma}$, where $\widehat{\Gamma}$ is a Zariski dense discrete subgroup of a connected positive dimensional semisimple complex Lie group $\widehat{S}$.

Proof: The proof proceeds by induction on $\operatorname{dim}_{\mathbb{C}} G / H$. The assertions are clear if $\operatorname{dim}_{\mathbb{C}} G / H=1$. Suppose first that $G^{\circ}$ is semisimple. If $H$ is an algebraic subgroup of $G$, then set $J=I:=H$ and we are done. If $H$ is not an algebraic subgroup of $G$, then let $J:=\bar{H}^{Z}$ be the Zariski closure of $H$ in $G$. If $J=G$, then set $I=H$. Since $G$ admits a faithful representation into some linear group, it follows that the 
normaliser $N_{G}\left(H^{\circ}\right)$, as the intersection of the image of $G$ with the normaliser of the image of $H^{\circ}$ in the linear group, is algebraic. The fact that $H \subset N_{G}\left(H^{\circ}\right) \subset G$, while $H$ is Zariski dense in $G$ implies $N_{G}\left(H^{\circ}\right)=G$. Because $H^{\circ}$ is solvable and $G^{\circ}$ is semisimple, this is impossible, unless $H^{\circ}=\{e\}$, that is, $H$ is discrete. If $J \neq G$, then $\operatorname{dim} J / B<\operatorname{dim} G / B$. By induction there are subgroups $H \subset I \subset J_{1} \subset J$ with $I^{\circ}$ solvable and $I^{\circ} \supset R_{J}, J_{1} / I=\widehat{S} / \widehat{\Gamma}$, where $\widehat{\Gamma}$ is a Zariski dense discrete subgroup of some semisimple complex Lie group $\widehat{S}$, and $J / J_{1}=S_{J} / A$, where $A$ is an algebraic subgroup of $S_{J}=J / R_{J}$. Since $\pi_{J}: J \rightarrow S_{J}$ is an algebraic map, $J_{1}=\pi_{J}^{-1}(A)$ is an algebraic subgroup of $G$ containing $H$ and contained in $J$. Hence $J_{1}=J$ and $G / H \rightarrow G / I \rightarrow G / J$ gives us the fibrations we seek.

In the rest of the proof assume $G^{\circ}$ is not semisimple, that is, $\operatorname{dim} R_{G}>0$. We assume first $\pi_{G}(H)$ is not closed in $S_{G}:=G / R_{G}$. Consider its closure $U:=\overline{\pi_{G}(H)}$ in $S_{G}$. Since $H^{\circ}$ is solvable, $U^{\circ}$ is also solvable by the result of Zassenhaus-Auslander, as proved in Theorem $\mathrm{A}$ in the Appendix of [11]. In general, $U$ is a real Lie group. Let $\widetilde{U}$ be the connected complex Lie subgroup of $S_{G}$ whose Lie algebra $\widetilde{u}$ is the complexification in the Lie algebra of $S_{G}$ of the Lie algebra $u$ of $U^{\circ}$. Note that $\widetilde{U}$ need not be closed. Let $N_{1}:=N_{S_{G}}(\widetilde{U})$ be the normaliser in $S_{G}$ of $\widetilde{U}$. Now $N_{1}$ is the intersection of the image of $S_{G}$ under a faithful representation into $G L(n, \mathbb{C})$ with the normaliser in $G L(n, \mathbb{C})$ of $\widetilde{U}$ and thus is an algebraic subgroup. By assumption $U^{\circ} \neq\{e\}$ and hence $\widetilde{U}$ has positive dimension. Thus $N_{1} \neq S_{G}$, since this latter group is semisimple and so the positive dimensional connected solvable subgroup $\tilde{U}$ cannot be normal in $S_{G}$. If $g \in U$, then $g \widetilde{U} g^{-1} \cap \widetilde{U}$ is a complex subgroup of $S_{G}$ containing $U^{\circ}$. By construction $\widetilde{U}$ is the smallest such subgroup, and thus $U \subset N_{1}$. Hence $\pi_{G}(H) \subset N_{1}$. Let $J_{1}:=\pi_{G}^{-1}\left(N_{1}\right) \subset G$. Note that $J_{1} \supset R_{G}$ and that $J_{1}$ has a finite number of connected components, because $N_{1}$ does and the fibres of the map $\pi_{G}$ are connected.

By recursion we assume $G \supset J_{i}$, where $J_{i} \supset H$ and $J_{i} \supset R_{G}$. We also assume that $N_{i}$ and thus $J_{i}$ are not solvable; see below. If the orbits of $R_{i}:=R_{J i}$ in $J_{i} / H$ are not closed, then we construct a closed complex subgroup $J_{i+1}$ of $G$ in the following way. Let $\pi_{i}: J_{i} \rightarrow S_{i}:=J_{i} / R_{i}$ denote the natural map. Note that $\operatorname{dim} R_{i}>\operatorname{dim} R_{G}>0$, where the first inequality follows from the fact that $N_{i}$ cannot be a semisimple Lie group, because it contains the positive dimensional normal solvable complex subgroup $\widetilde{U}_{i-1}$. The group $\widetilde{U}_{i}$ is the complexification in $S_{i}^{\circ}$ of the identity component (which, by the Zassenhaus-Auslander result, is again solvable) of the closure of $\pi_{i}(H)$. The normaliser $N_{i+1}$ of $\tilde{U}_{i}$ in $S_{i}$ is a proper algebraic subgroup of $S_{i}$ containing $\pi_{i}(H)$ and we set $J_{i+1}:=\pi_{i}^{-1}\left(N_{i+1}\right)$. Note that $J_{i+1} \supset R_{i} \supset R_{G}$. If the subgroup $N_{i+1}^{\circ}$ is solvable, then we set $I=J:=J_{i+1}$ and this is the group we seek. For, in this case it is clear $I^{\circ}$ is solvable. The fact that $G / J$ is the quotient of $S_{G}$ by an algebraic subgroup 
is shown below in the last paragraph of the proof. The Klein form of $J / I$ is obvious in this case.

Otherwise, since $\operatorname{dim}_{\mathbb{C}} G / H$ is finite, the process described above terminates after finitely many steps yielding a chain of closed complex subgroups $J_{i}$, for $1 \leqslant i \leqslant k$, of $G$ with

$$
H \subset J_{k} \subset J_{k-1} \subset \cdots \subset J_{1} \subset G,
$$

such that the $R_{k}$-orbits are closed in $J_{k} / H$ and thus in $G / H$ too. Each $J_{i}$ has a finite number of connected components, since it is the inverse image of an algebraic subgroup by a map with connected fibres. In addition, $J_{i} / J_{i+1}=S_{i} / N_{i+1}$, where $S_{i}:=J_{i} / R_{i}$ and $N_{i+1} \subset S_{i}$ is an algebraic subgroup, given by the above procedure. Because $H$ is a subgroup of $J_{k}$ and $R_{k}$ is normal in $J_{k}$, it follows that $H \cdot R_{k}$ is a closed subgroup of $J_{k}$. Incidentally, note that if $\pi_{G}(H)$ is closed in $S_{G}$ to begin with, then we set $J_{k}:=G$ and the above properties are trivially verified. The projection $H_{k}:=\pi_{k}(H)$ is closed in $S_{k}:=J_{k} / R_{k}$ and $H_{k}^{\circ}$ is solvable. Since $S_{k}^{\circ}$ is semisimple, it follows from the first paragraph of the proof that there exist a closed complex subgroup $I_{S}$ with $I_{S}^{\circ}$ solvable and an algebraic subgroup $A_{S}$ with $H_{k} \subset I_{S} \subset A_{S} \subset S_{k}$ such that $A_{S} / I_{S}=\widehat{S} / \widehat{\Gamma}$ is the quotient of a semisimple complex Lie group $\widehat{S}$ by a Zariski dense discrete subgroup $\widehat{\Gamma}$, unless $I_{S}=A_{S}$. Now set $I:=\pi_{k}^{-1}\left(I_{S}\right)$ and $J:=\pi_{k}^{-1}\left(A_{S}\right)$. Because $\pi_{k}(H)=H_{k} \subset I_{S} \subset A_{S}$, it follows that $H \subset I \subset J$. Since $I^{\circ}=\pi_{k}^{-1}\left(I_{S}^{\circ}\right)$ is the inverse image of a solvable group by a homomorphism with solvable kernel, $I^{\circ}$ is solvable. Also

$$
J / I=A_{S} / I_{S}=\widehat{S} / \widehat{\Gamma},
$$

and we see from (1) that $J / I$ has the required Klein form.

Since $J \supset R_{G}$, it is clear $G / J=S_{G} / \pi_{G}(J)$, where $\pi_{G}(J)$ is a closed complex subgroup of $S_{G}$. We would like to show that $\pi_{G}(J)$ is an algebraic subgroup of $S_{G}$, that is, that one can write $G / J$ as a quotient of $S_{G}$ by an algebraic subgroup. We have already defined the natural map $\pi_{i}: J_{i} \rightarrow S_{i}:=J_{i} / R_{i}$ and we also consider the natural map

$$
\widehat{\pi}_{i}: N_{i} \rightarrow N_{i} / R_{N_{i}} \text {. }
$$

Note that $R_{N_{i}}=\pi_{i-1}\left(R_{i}\right)$ and thus $S_{i}=\widehat{\pi}_{i}\left(N_{i}\right)$. For any $1 \leqslant i \leqslant k-1$ we have the following diagram

$$
\begin{array}{rrrrr}
J_{i+1} & :=\pi_{i}^{-1}\left(N_{i+1}\right) & \subset J_{i} \\
\pi_{i+1} \downarrow & & & \downarrow \pi_{i} \\
S_{i+1} & \stackrel{\widehat{\pi}_{i+1}}{\longleftarrow} N_{i+1} & \subset S_{i}
\end{array}
$$


and thus

$$
\pi_{i+1}=\left.\widehat{\pi}_{i+1} \circ \pi_{i}\right|_{J_{i+1}}
$$

There is a similar diagram for $i=0$, if one replaces $J_{0}$ by $G, S_{0}$ by $S_{G}$ and $\pi_{0}$ by $\pi_{G}$. Then

$$
\pi_{1}=\left.\widehat{\pi}_{1} \circ \pi_{G}\right|_{J_{1}}
$$

We now define a group

$$
A:=\widehat{\pi}_{1}^{-1} \circ \cdots \circ \widehat{\pi}_{k-1}^{-1} \circ \widehat{\pi}_{k}^{-1}\left(A_{S}\right) \subset S_{G}
$$

Note that $A$ is an algebraic group, because $A_{S}$ is algebraic and each of the maps $\hat{\pi}_{i}$ is an algebraic map. Now by using (2) and (3) we see that

$$
\begin{aligned}
J & =\pi_{k}^{-1}\left(A_{S}\right) \\
& =\pi_{k-1}^{-1} \circ \widehat{\pi}_{k}^{-1}\left(A_{S}\right) \\
& =\cdots \\
& =\pi_{G}^{-1} \circ \widehat{\pi}_{1}^{-1} \circ \widehat{\pi}_{2}^{-1} \circ \cdots \circ \widehat{\pi}_{k}^{-1}\left(A_{S}\right) \\
& =\pi_{G}^{-1}(A) .
\end{aligned}
$$

Hence $\pi_{G}(J)=A$ and thus $G / J=S_{G} / A$. If the process ended because $N_{k}^{\circ}$ is solvable, then we set

$$
A:=\widehat{\pi}_{1}^{-1} \circ \cdots \circ \widehat{\pi}_{k-1}^{-1} \circ \widehat{\pi}_{k}^{-1}\left(N_{k}\right) \subset S_{G} .
$$

In this case $A$ is algebraic for the same reasons as before and $A^{\circ}$ is also solvable. Similar to above, it follows that $G / J=S_{G} / A$ and the proof of the theorem is complete.

\section{The structure of arbitrary $G / H$}

In conclusion we would like to point out how the structure of any homogeneous complex manifold $G / H$ depends on six components. First let $N:=N_{G}\left(H^{\circ}\right)$ be the normaliser in $G$ of the connected component $H^{\circ}$ of the identity of $H$. Then $N$ is a closed complex subgroup of $G$ containing $H$ and one has the fibration $G / H \rightarrow G / N$ which is commonly called the normaliser fibration of $G / H$. By a Theorem of Chevalley [3] the commutator subgroup $G^{\prime}$ of $G$ has closed orbits in $G / N$ and one has the fibration $G / N \rightarrow G / N G^{\prime}$. Its base $G / N G^{\prime}$ is a Stein Abelian Lie group, see [7], and its fibre $N G^{\prime} / N=G^{\prime} / N \cap G^{\prime}$ can be written as a quotient of algebraic groups. Since the radical $R_{G^{\prime}}$ of the commutator subgroup $G^{\prime}$ has closed orbits in $G^{\prime} / G^{\prime} \cap N$, one can consider the fibration

$$
G^{\prime} / G^{\prime} \cap N{\stackrel{R_{G^{\prime}} / R_{G^{\prime}}}{\longrightarrow}}^{\cap N} S / S \cap N R_{G^{\prime}}
$$


where $S$ denotes a maximal semisimple subgroup of $G$. As well, the fibre $N / H$ of the normaliser fibration can be handled by the theorem above, because $N / H=$ $\left(N / H^{\circ}\right) /\left(H / B^{\circ}\right)$ is the quotient of the complex Lie group $N / H^{\circ}$ by the discrete subgroup $H / H^{\circ}$. Thus one can study $G / N G^{\prime}, R_{G^{\prime}} / R_{G^{\prime}} \cap N$ and $S / S \cap N R_{G^{\prime}}$ along with the three components of $N / H$ given by our structure theorem. Variants of these ideas can be found in many different works in the literature, for example, see [4]. There it sufficed to know that if $G$ is a mixed complex Lie group (neither solvable nor semisimple) and $\Gamma$ is a discrete subgroup of $G$, then there exists a proper closed complex subgroup $J$ of $G$ containing both $\Gamma$ and the radical $R_{G}$ and one then has the fibration $G / \Gamma \rightarrow G / J$ to work with.

\section{REFERENCES}

[1] L. Auslander, 'On radicals of discrete subgroups of Lie groups', Amer. J. Math. 85 (1963), 145-150.

[2] L. Auslander and R. Tolimieri, 'Splitting theorems and the structure of solvmanifolds', Ann. of Math. 92 (1970), 164-173.

[3] C. Chevalley, Théorie des groupes de Lie II: Groupes algébriques (Hermann, Paris, 1951).

[4] B. Gilligan, 'Ends of complex homogeneous manifolds having non-constant holomorphic functions', Arch. Math. 37 (1981), 544-555.

[5] B. Gilligan and P. Heinzner, 'Globalization of holomorphic actions on principal bundles', (preprint, 1995).

[6] V. V. Gorbatsevich and A. L. Onishchik, 'Lie groups of transformations', (in Russian), in Current problems in mathematics, Fundamental directions. 20, pp. 103-238, Moscow, 1988; Engl. transl. in Enclyclopaedia of Mathematical Sciences, 20 (Springer-Verlag, 1993) 95-229.

[7] A. T. Huckleberry and E. Oeljeklaus, 'Homogeneous spaces from a complex analytic viewpoint', in Manifolds and Lie groups. (Papers in honor of Y. Matsushima), Progress in Math. (Birkhäuser, Boston, 1981), pp. 159-186.

[8] F.I. Karpelevich, 'On a fibration of homogeneous spaces', (in Russian), Uspekhi Mat. Nauk 11 (1956), 131-138.

[9] G.D. Mostow, 'On covariant fiberings of Klein spaces, I, II', Amer. J. Math. 77 (1955), 247-278; 84 (1962) 466-474.

[10] G.D. Mostow, 'Some applications of representative functions to solv-manifolds', Amer. J. Math. 93 (1971), 11-32.

[11] H-C. Wang, 'On the deformations of lattices in a Lie group', Amer. J. Math. 85 (1963), 189-212.

Department of Mathematics and Statistics

University of Regina

Regina

Canada S4S 0A2

e-mail: gilligan@max.cc.uregina.ca 